\title{
Intelligent Greedy Pursuit Model for Sparse Reconstruction Based on $l_{0}$ Minimization
}

\author{
Dan Li, Qiang Wang Member IEEE, Yi Shen Member IEEE ${ }^{1}$
}

Harbin Institute of Technology, Control Science and Engineering,

No.92 West Da-Zhi Street, Nangang District, Harbin, China, 150001

lidanhit@163.com,wangqiang@hit.edu.cn,shen@hit.edu.cn

\begin{abstract}
$l_{0}$ minimization based sparse reconstruction is an NP-hard problem with very high computational complexity, which is difficult to be achieved by traditional algorithms. Although greedy algorithm aims at solving $l_{0}$ minimization, it is more likely to obtain a sub-optimal solution. In this paper, we propose an intelligent greedy pursuit (IGP) algorithm to solve the $l_{0}$ minimization essentially. Firstly, we propose a novel optimization function for the sparse reconstruction problem with the sparsity level unknown as a prior. Then, a two-cycle optimization algorithm is designed, whose object is to estimate the support collection and its corresponding coefficients intelligently and accurately by searching for the global optimal solution. To this end, we take advantage of intelligent optimization algorithm in global searching and solving combinatorial optimization problems to guide the intelligent estimation. Also, the principle of estimation is designed by the matching strategies of greedy algorithm which performs quite well in reconstruction speed. The so-called IGP model is as simple as greedy algorithm, while it has been proved through experiments that the performance of IGP for signal reconstruction and image reconstruction outperforms the state-of-the-art reconstruction algorithms. Moreover, IGP can reconstruct signal accurately with a relatively small measurement rate.

Keywords: Compressive sensing; $l_{0}$ minimization; Intelligent optimization algorithm; Greedy algorithm.
\end{abstract}




\section{Introduction}

In compressive sensing (CS) theory [1? ], if the original signal is sparse or compressed, it can be reconstructed with high quality from its degraded version. Assume $x$ is a signal with size of $N * 1$ and it can be represented sparsely in the sparse basis $\psi$. Given a measurement matrix $\phi$ with size of $M * N$ (where $M<N$ ) which is unrelated to the sparse basis $\psi$ and satisfies the restrict isometry property (RIP). The measurement signal $y$ can be obtained by Equ.(1).

$$
y=\phi * x=\phi * \psi * s
$$

where, $s \in \mathbf{R}^{\mathbf{N}}$ is a sparse signal.

Reconstruction [2] is a process of recovering the high-dimensional sparse signal $s$ from the low-dimensional measurement signal $y$. To simplify the problem, It is assumed that $x$ is sparse in this paper and that $x$ and $s$ can be used interchangeably. Mathematically, the reconstruction attempts to recover $x$ by solving the $l_{0}$ minimization problem of the form $x=\operatorname{argmin}\|x\|_{0}$ subject to $y=\phi * x$, where $\|x\|_{0}$ is the $l_{0}$ norm of $x$ and is measured from the number of nonzero elements. This optimization problem means that we need to find a solution which has the least non-zero elements from all the solutions which satisfy the equation $y=\phi * x$. Then, this problem can be generally formulated in the following Lagrangian form.

$$
x=\operatorname{argmin}\|x\|_{0}+\lambda *\|y-\phi * x\|_{2}^{2},
$$

where $\lambda$ is a regularization parameter and an appropriate $\lambda$ can make a good balance between the estimation error and sparsity level.

$l_{0}$ minimization in Equ.(2) is an NP-hard problem that requires exhaustively listing all possibilities of the original signal and is difficult to be achieved by traditional algorithms. Also, $l_{0}$ minimization is nonconvex, so we always transform Equ.(2) to Equ.(3). 


$$
x=\operatorname{argmin}\|x\|_{1}+\lambda *\|y-\phi * x\|_{2}^{2},
$$

The problem in Equ.(3) is convex and methods available for Equ.(3) are convex optimization algorithms $[3,4]$, such as basis pursuit algorithm $[5,6]$ and linear programming algorithm [7]. However, a fact that is often neglected is, the conditions guaranteeing the equivalence of $l_{0}$ minimization and $l_{1}$ minimization are not necessarily satisfied.

To solve Equ.(2) directly, many efficient greedy algorithms are developed, such as Orthogonal matching pursuit algorithm (OMP) [8, 9], subspace pursuit algorithm (SP) [10], Polytope Faces Pursuit algorithm (PFP) [11], compressive sampling matching pursuit (CoSaMP)[12], Primal-Dual Barrier Method (PDCO) [13], backtracking-based matching pursuit (BAOMP) [14], Sparsity Adaptive Matching Pursuit algorithm (SAMP)[15] and stagewise orthogonal matching pursuit (StOMP) [16]. Greedy algorithm aims at estimating the support collection and its corresponding coefficients of the original signal iteratively based on the residual correlation. However, the residual correlation can just reflect the connected degree between the coefficients and the measurement signal but can not indicate that the bigger the residual correction is, the bigger the nonzero coefficient is. Although greedy algorithm achieves satisfactory reconstruction quality under specific conditions, it performs better only when the sparsity level is relatively small or the measurement rate is relatively large. Also, the sparsity level is always known as a prior in greedy algorithm, which leads to a strong limitation as the sparsity level is hard to be estimated in many reconstruction problems. Moreover, all the greedy algorithms adopt suboptimal fast searching strategies, so they are easy to fall into the suboptimal solutions.

Intelligent optimization algorithm (IOA) $[17,18,19]$ is famous for its efficient global searching strategies and superior performance in solving nonconvex problems and combinatorial optimization problems. While the $l_{0}$ minimization is a combinatorial optimization problem, it is quite natural to consider employing IOA to solve the optimization problem in Equ.(2). Two methods [20, 21] based 
on simulated annealing algorithm are proposed for signal reconstruction and they had been proved to perform well in searching global optimization solution. However, the two methods are both single-cycle algorithm, which may obtain a local optimal solution when the measurement number is relatively small. Also, the precondition of $[21,22]$ is that the sparsity level must be known as a prior, which limits the applications as the sparsity level is always unknown in practice, such as the sparsity level is hard to be estimated in image reconstruction. In [22], the authors establish an evolutionary multi-objective method for sparse reconstruction. An adaptive recovery method [23] based on compressive sensing and genetic algorithm is proposed for image reconstruction, in which the necessary measurements and the computational complexity are high. In our previous work $[24,25]$, we propose two signal reconstruction algorithms based on the combination of genetic algorithm and bacterial foraging optimization algorithm (GA-BFO) and artificial immune algorithm (AIA) respectively, in which the sparsity level is also necessary as a prior and the computational complexity is high. So how to utilize the advantages of intelligent optimization algorithm and design an optimal strategy is still a big challenge.

In this paper, we propose an intelligent greedy pursuit (IGP) model which aims at solving the $l_{0}$ minimization essentially for CS reconstruction. The objective of IGP model is to estimate the support collection of the original signal and its corresponding coefficients intelligently and accurately without knowing the sparsity level as a prior. The proposed IGP model includes two aspects: the modeling of IGP and the algorithm of IGP. We first propose a novel optimization function for the sparse reconstruction with the sparsity level unknown in advance, and then develop a two-cycle optimization algorithm to solve the optimization problem. In the two-cycle optimization algorithm, the aim is to estimate the collection which contains the support collection and its corresponding values intelligently. As intelligent optimization algorithm is efficient in searching for the global optimal solution, it is introduced to guide the intelligent estimation and improve the performance of reconstruction. The principle of estimation is designed by the matching strategies of greedy algorithm which 
performs better in reconstruction speed. The two-cycle optimization problem is superior in both global searching and local searching, so it can not only avoid falling into a sub-optimal solution but also improve the reconstruction speed. It is the first time that the IGP model is employed to solve the nonconvex $l_{0}$ minimization problem essentially, which makes the global optimal solution can be accurately estimated possible. The proposed intelligent greedy pursuit (IGP) model is as simple as greedy algorithm, while it can find the global optimal solution and is superior to greedy algorithm on reconstruction quality, such as OMP, PFP, CoSaOMP , SAMP and BAOMP. Extended simulations conducted on typical reconstruction problems, including signal reconstruction and image reconstruction, demonstrate that the proposed model IGP outperforms the state-of-the-art reconstruction methods, including both convex optimization algorithms and greedy algorithms. Moreover, another significant improvement is that IGP can reconstruct signal accurately when the measurement rate is relatively small or the sparsity level is relatively large.

The major contributions of this paper are fourfold:

1. We propose a novel optimization function for the sparse reconstruction which does not require the sparsity level known as a prior.

2. We develop a two-cycle optimization algorithm by taking advantage of intelligent optimization algorithm in global optimization problems and utilizing the superior performance of greedy algorithm in reconstruction speed to solve the $l_{0}$ minimization essentially.

3. The proposed model IGP can reconstruct signal accurately with a relatively small measurement rate or a relatively large sparsity level.

4. The proposed model IGP is as simple as greedy algorithm, while it overcomes the common shortcoming that greedy algorithm is more likely to fall into a sub-optimal solution.

The rest of this paper is organized as follow: Section 2 presents the modelling of IGP, the two-cycle optimization algorithm of IGP, the computational 
complexity analysis and connections between IGP and other existing works. Simulation results are given in Section 3 to illustrate the performance of the proposed model IGP. Section 4 concludes this paper.

The follow notations are used in the rest of this paper. The $l_{0}$ norm of signal $x$ can be represented by $\|x\|_{0}$. Let $\phi$ indicates the measurement matrix and $\phi_{i}$ represents the $i t h$ column of $\phi . S=1,2, \ldots, N$ indicates the index of each entries of the original signal, where $N$ represents the length of the original signal. Suppose $K$ entries are selected from $S$ to generate the support collection $I_{K}$, the vector $x_{I_{K}}$ consists the entries of $x$ indexed by $i \subset I_{K}$ and the matrix $\phi_{I_{K}}$ is composed of the columns of $\phi$ with indexes $i \subset I_{K}$. The pseudo-inverse operation of measurement matrix $\phi$ can be calculated by $\phi^{\dagger}=\left(\phi^{\top} * \phi\right)^{-1} * \phi^{\top}$, where $T$ represents matrix transportation.

\section{Intelligent Greedy Model}

In this section, we first propose a novel optimization function for the sparse reconstruction problem, and then design a two-cycle optimization algorithm to solve the proposed optimization function. Next, we analyse the computational complexity of IGP. In the end, we compare our IGP model to some existing arts.

\subsection{Modeling of IGP}

Suppose the original signal $x$ is sparse and the sparsity level $K$ is known as a prior, we can obtain the measurement signal by $y=\phi * x$, where $\phi$ is the measurement matrix. As applied in many greedy algorithms such as OMP, $\mathrm{SP}$, and CoSaMP, the $l_{0}$ minimization problem can be handled by a two-step strategy. In the first step, an estimated support collection $I$ which satisfies $\|x\|_{0}=K$ is obtained. Then the nonzero elements of the reconstructed signal recx $_{I}$ can be calculated using the least square method and the estimated solution recx is obtained by Equ.(4) in the second step.

$$
\operatorname{recx}_{I}=\phi_{I}^{\dagger} * y \quad \text { and } \quad \operatorname{recx}_{S-I}=0 .
$$


However, the sparsity level is hard to be estimated in many reconstruction problem in practice. So we propose a novel optimization function in Theorem 1 when the sparsity level is unknown in advance. Before giving it, we provide a uniqueness solution method in Lemma proposed in [21], which can be used to prove the Theorem 1.

Lemma: Suppose that recx* with support collection $I^{*}$ is the unique solution of Equ.(2), and that the sparsity level satisfies $K<\operatorname{spark}(\phi) / 2$, where $\operatorname{spark}(\phi)$ is the smallest number of columns from $\phi$ that are linearly dependent. Then we have $r e c x=r e c x^{*}$ if and only if $\phi_{I^{*}} * \phi_{I^{*}}^{\dagger} * y=y$, where $I^{*}$ satisfying $I^{*}=K$ is the estimated support collection and the estimated solution $r e c x^{*}$ is obtained using Equ.(4).

As described in Lemma, if the sparsity level satisfies $K<\operatorname{spark}(\phi) / 2$, we can obtain the unique solution by seeking a support collection $I$ which satisfies $I=K$ and $\phi_{I} * \phi_{I}^{\dagger} * y=y$. If the estimated support collection can be estimated, it satisfies $F(I)=0$. As Equ.(5) satisfies $f(I) \geq 0$, the optimization function of [21] can be set as Equ.(5) and the estimated support collection can be obtained by optimizing Euq.(6) when the sparsity level is known as a prior.

$$
\begin{gathered}
f(I)=\left\|\phi_{I} * \phi_{I}^{\dagger} * y-y\right\|_{2}^{2}, \\
\min _{\in \Theta} f(I) .
\end{gathered}
$$

Theorem 1: Suppose the sparsity level $K$ which satisfies $K \leq \operatorname{spark}(\phi) / 2$ is unknown in advance, the two-step strategy turns to estimate the collection $I_{m m}$ by optimizing the optimization function described in Equ.(7) and then the estimated solution can be obtained by Equ.(8).

$$
\min _{I_{m m}} f\left(I_{m m}\right)=\left\|\phi_{I_{m m}} * \phi_{I_{m m}}^{\dagger} * y-* y\right\|_{2}^{2},
$$

where, the collection $I_{m m}$ represents all $m m$ - cardinality (where $m m$ is bigger than the sparsity level $K$ and smaller than the measurement number $m$ ) subsets 
of the set $S$.

$$
\operatorname{recx}_{I_{m m}}=\phi_{I_{m m}}^{\dagger} * y \quad \text { and } \quad \operatorname{recx}_{S-I_{m m}}=0
$$

Proof: As proved in [16], let the columns of $\phi$ be in general position, suppose $I_{0}$ denotes the support of the original signal $x_{0}$ and $I_{s}$ denotes the support of $x_{s}$. If $I_{0}$ is a subset of $I_{s}$, then we have perfect recovery:

$$
x_{s}=x_{0}
$$

If the support collection $I$ satisfies $\left\|\phi_{I} * \phi_{I}^{\dagger} * y-y\right\|_{2}=0$ and the collection $I_{m m}$ includes $I, I_{m m}$ must satisfy the condition that $\left\|\phi_{I_{m m}} * \phi_{I_{m m}}^{\dagger} * y-y\right\|_{2}=0$. So Equ.(7) can be set as the optimization function and the reconstructed signal can be obtained by optimizing the problem $\min f\left(I_{m m}\right)$ when the sparsity level is unknown in advance.

The representation of the optimization function of IGP is the same as that of the optimization function in [21]. However, the independent variable $I_{m m}$ of the optimization function of IGP is different to that $I$ of the optimization function in [21], which is the main difference between them. The length of $I_{m m}$ doesn't equal to the sparsity level, which means the sparsity level need not to be known as a prior for reconstruction. The proposed optimization function of IGP can relax the initial precondition for reconstruction and can extend the applications of IGP.

In the condition that the sparsity level is known as a prior, the size of the feasible domain $\Theta$, which represents the solution set consisted of all $\mathrm{K}$ cardinality subsets of the set $S$, can be calculated by $C_{N}^{K}$. However, there is only one global optimal solution. As mentioned in Theorem 1, the size of the feasible domain $\Theta^{\prime}$ can be calculated by $C_{N}^{m m}$ when the sparsity level is unknown as a prior. While there are $C_{N-K}^{m m-K}$ global optimal solutions. The main reason is that the number of estimated $I_{m m}$ which satisfies $I \in I_{m m}$ can be calculated by $C_{N-K}^{m m-K}$. So the proposed method IGP can find the optimal solution more easily than the situation that the sparsity level is known as a prior 
for many reconstruction problems.

\subsection{Algorithm of IGP}

Intelligent optimization algorithm (IOA) is a kind of random searching algorithm achieved by simulating natural selection and genetic variation, which is inspired by biological evolution. IOA is famous for its success in solving many complex problems, including combinational optimization problems and NP-hard problems such as Traveling Salesman Problem (TSP) [26, 27]. Each individual in the group represents a possible solution and the solution set of the optimization function is considered as the searching area. There are three classical and effective operations, i.e. clone, mutation and selection. The clone operation generates more individuals, which is beneficial for searching simultaneously. The mutation scheme introduces new individuals to increase the diversity of the group, which is conducive to be more closer to the global optimal solution. The selection operation can choose and save the best individual. After the three intelligent operations, the individuals is more likely to convergent to the global optimal solution.

In this section, we design a two-cycle optimization algorithm for the proposed optimization function in section 2.1. The aim of the two-cycle algorithm is to estimate the collection $I_{m m}$ which contains the support collection of the original signal intelligently and accurately. Based on the simultaneous searching of all the individuals, the outer loop of the two-cycle scheme is superior in global searching, which makes the algorithm more likely to find the global optimal solution. However, an obvious shortcoming is that the process of outer loop is time-consuming in practical application. The inner loop of the two-cycle algorithm which is in charge of local searching is designed to accelerate the reconstruction speed. The inner loop is an essentially iterative random searching procedure and can select a new solution from the neighbourhood of the current solution. Based on the Metropolis rule, the inner loop permits accepting a less optimal solution and also some random solutions with positive probability, which can also avoid falling into a sub-optimal solution. 
The proposed two-cycle optimization algorithm has both strong global searching ability and strong local searching ability. Nevertheless, the computational complexity of the two-cycle optimization scheme is high, which leads to a slow reconstruction speed. Considering the superior performance of greedy algorithm in reconstruction speed, the estimation principle of the two-cycle scheme is designed by the matching strategies of greedy algorithm. The estimation principle in the outer loop is designed using the selection strategy in OMP and that in the inner loop is designed using the pruning technique in CoSaMP. The parallel searching of the individuals also can reduce the computational complexity. In the end, the proposed so-called intelligent greedy pursuit algorithm is as easy as the greedy algorithm which aims at finding the estimated support collection of the original signal.

The Algorithm of IGP based signal reconstruction is shown in Algorithm 1, where each individual represents an estimated solution $I_{m m}$ and $f\left(I_{m m}\right)=\|$ $\phi_{I_{m m}} * \phi_{I_{m m}}^{\dagger} * y-* y \|_{2}^{2}$ is set as the fitness function. The details of the main steps of Algorithm 1 are described as follows.

1) Initialization. The individuals of the initial group are generated using the matching strategy of OMP algorithm and SP algorithm. Firstly, calculate $\phi^{\dagger} * y$ and select the $m m$ bigger indexes as the first individual. Then, remove the biggest index $i_{1}$, calculate $\phi_{S-i_{1}}^{\dagger} * y$ and select the $m m$ bigger indexes as the second individual. The same for other initial individuals.

2) Searching strategy and estimation principle of the outer loop. Firstly, calculate the fitness of each individual based on the optimal function $f\left(I_{m m}\right)=\|$ $\phi_{I_{m m}} * \phi_{I_{m m}}^{\dagger} * y-* y \|_{2}^{2}$ and save the indexes $I_{i}^{0}$ of the highest value for each individual. Then, combine all the $I_{i}^{0}$ to be the bestindividual. After the selection operation, mutation is implemented to the group. The mechanism of mutation operation is: (1) calculate $x_{I_{m m}}$ for each individual based on $x_{I_{m m}}=\phi_{I_{m m}}^{\dagger} * y$; (2) remain the indexes whose numerical value are bigger and select randomly from $S-I$ to take place the smaller ones. After the mutation operation, a new group which is closer to the global optimal solution is generated.

3) Searching strategy and estimation principle of the inner loop. The esti- 


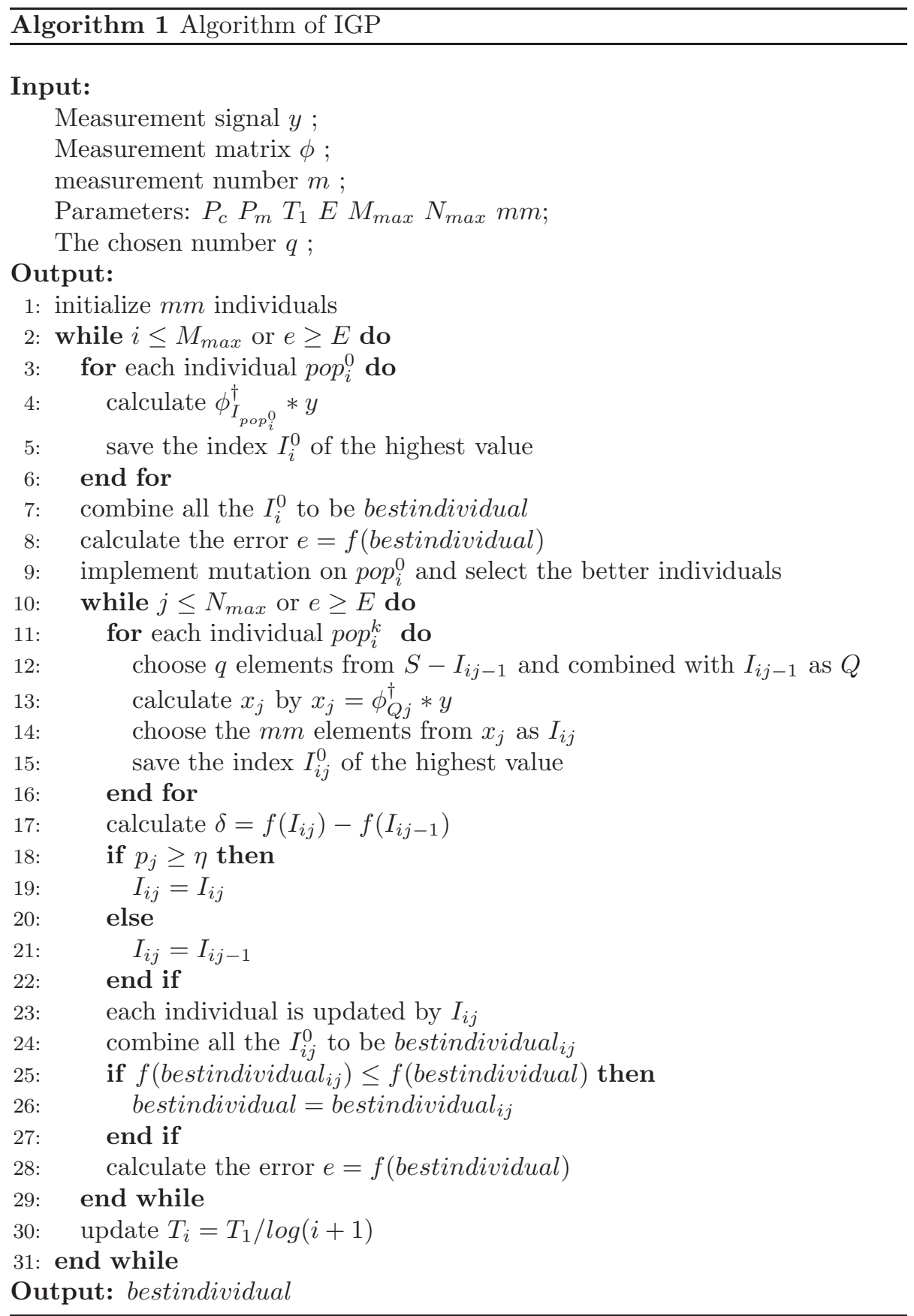


mation strategy of the inner loop is implemented on each individual generated in the outer loop. Firstly, select $q$ elements from $S-I_{i j-1}$ and combined them with $I_{i j-1}$ to generate $Q$. Secondly, calculate $x_{Q}=\phi_{Q}^{\dagger} * y$ and choose the $m m$ bigger elements from $x_{Q}$ as the new generated individual. Thirdly, calculate the fitness of the new individual and the old individual and select one individual based on Metropolis rule. Metropolis rule is that: if $p \leq \eta$, where $p$ is calculated by Equ.(10) and $\eta$ is a random number chosen in $[0,1]$, select the new individual, otherwise, select the old individual.

$$
p=\min \left(1, \exp \left(-\left(f\left(I_{i j}\right)-f\left(I_{i j-1}\right)\right) / T_{i}\right)\right) .
$$

4) Selection. Combine all the indexes $I_{i j}^{0}$ to be bestindividual ${ }_{i j}$ and compare it to the bestindividual, if bestindividual ${ }_{i j} \leq$ bestindividual, implement bestindividual $=$ bestindividual $_{i j}$. The parameter which is calculated by $T_{i}=T_{1} / \log (i+1)$ is updated in the outer loop.

5)Terminal condition. The terminal condition is that the number of iterations reach the maximum number or the error is smaller than the minimum value $E$.

After the intelligent estimation of the two-cycle scheme, the obtained bestindividual represents the estimated collection $I_{m m}$ which contains the support collection of the original signal. Then the reconstructed signal can be obtained by Equ.(8).

\subsection{Computational complexity analysis of IGP}

As we can see from Algorithm 1, the main computational complexity of the proposed model lies in the initialization and the iterations. While the main computational complexity of the iterations lies in step 4 in the outer loop and step 13 in the inner loop. The computational complexity of the initialization can be calculated by $\sum_{i=0}^{m m-1} O(N-i)^{3}$. Step 4 and step 13 both have matrix multiplication and its computational complexity is upper bounded by $O\left(N^{3}\right)$. In each iteration, a total of $m m$ individuals compute simultaneously . Also, the maximum number of iterations can be calculated by $M_{\max } * N_{\max }$. So the computational complexity of IGP can be estimated by Equ.(11). 


$$
\sum_{i=0}^{m m-1} O(N-i)^{3}+m m * M_{\max }\left(N_{\max }+1\right) * O\left(N^{3}\right)
$$

From Equ.(11), we can see that the size of searching individuals and the iterations influence the computational complexity significantly. However, the parallel computation of the searching individuals can reduce the iterations significantly. So if we want to reduce the computational complexity of IGP model in the future, some methods can be introduced to optimize the initial solution, which is beneficial to reduce the iterations.

\subsection{Connections between IGP and existing works}

In our previous work $[24,25]$, we propose two reconstruction methods based on GA-BFO and artificial immune algorithm (AIA) by solving $l_{0}$ minimization. Although the two methods in $[24,25]$ achieve superior performance in reconstruction quality, the computational complexity of them are high as no updating mechanism of greedy algorithm is introduced. In [21] and [28], a hybrid SA thresholding algorithm (HSAT) and a simulated annealing algorithm are developed for signal reconstruction (SASR), which are both proposed in the framework of SA. However, our method IGP is very different from them. There are two main differences between the proposed IGP and the existing works. As step 11 to step 14 of the proposed two-cycle algorithm are much similar to the updating strategies of SASR, the proposed two-cycle algorithm is more likely a swarm version of SASR. In the swarm version, we also design the advance estimation principles in both the inner loop and the outer loop which are very different to that of the existing works, such as the estimation principle of IGP is designed using the matching strategies of parallel OMP and CoSaMP, whereas that in SASR is designed using SP. Also, the initialization of IGP is designed by the strategies of parallel OMP and SP, whereas that in SASR is designed by the q-thresholding algorithm. The two-cycle algorithm of the proposed IGP model makes the individuals searching parallelly in both the outer loop and the inner loop, which not only can make it more likely to find the global optimal 
solution even though the measurement number is relatively small but also can reduce the iterations to accelerate the reconstruction speed. The swarm version and the estimation principle of the two-cycle algorithm are both the main differences between the proposed IGP and the existing works. Moreover, another main difference between the proposed IGP model and the existing works is that IGP doesn't need the sparsity level known as a prior. IGP model proposes a novel optimization optimization function for the sparse reconstruction with the sparsity level unknown in advance, which can relax the initial precondition of reconstruction and extend the application of IGP.

After the above analysis, our proposed IGP method is very different to the existing works. The global searching ability of IGP is superior to that of the existing algorithms and the reconstruction speed of IGP is also improved. Also, the optimization function without knowing the sparsity level as a prior can extend the applications of IGP model.

\section{EXPERIMENT RESULTS AND ANALYSIS}

\subsection{Experimental Configuration}

In this section, CS reconstruction experiments on signal reconstruction and image reconstruction are conducted to verify the reconstruction performance of IGP model. The measurement matrix $\phi$ is obtained by extracting $m$ rows of the $N * N$ orthogonal random matrix. The proposed IGP model is compared with those state-of-the-art greedy algorithms of OMP, PFP, SAOMP, CoSaOMP and BAOMP for signal reconstruction and those methods of BP, Lasso [29], OMP, PFP, tree-CoSaMP [30] and edge based matching pursuit algorithm (EMPA) [31] for image reconstruction. In the experiments of image reconstruction, a block sampling scheme $[32,33]$ is utilized to improve the reconstruction speed, that is, the original images are blocked by windows with the size of $32 * 32$. We obtain the measurements from the subblocks and reconstruct these subblocks respectively. We also set the main parameters $M_{\max }=200, N_{\max }=200$, 
$E=10^{-5}$ and the chosen number $q=\operatorname{round}(0.2 * m)+1$ of our algorithm for all experiments. And then the parameter $m m$ can be calculated by $m m=m-q$.

All the experiments are implemented in Matlab 2011a on the PC with 3.2GHZ Intel Core $i 5$ processor and 8.0GB memory running the Windows 7 system. Here, the compared algorithms OMP, PFP and BP are implemented using SolveOMP, SolvePFP and SolveBP in SparseLab toolbox [34] respectively.

\subsection{Experiments on signal}

In this experiment, the test signal is sparse with the Gaussian random nonzero coefficients. The process of reconstruction is supposed to be noiseless. Considering the random property of IGP, all simulation results are obtained by averaging the results of 500 independent tests. The two evaluation indexes are exact reconstruction rate and average runtime respectively. Exact reconstruction rate is calculated as the percentage of the exact reconstruction from the 500 tests and average runtime is calculated by averaging the runtimes of the exact tests.

Firstly, we fix $N=50$ and $K=5$ and change the measurement number $m$ from 5 to 30. The reconstruction performance of the six methods are evaluated by the exact reconstruction rate and average runtime, which are shown in Fig.1. From Fig.1.(a), we can find that our method IGP achieves the best reconstruction quality for all the measurement number. Also, IGP is significantly superior to the state-of-the-art greedy algorithms when the measurement number is relatively small, even that the measurement number is just a little larger than the sparsity level. Considering the average runtime performance of the six methods, Fig.1.(b) shows that OMP has the fastest reconstruction speed and IGP method is a little slower than the state-of-the-art greedy algorithms. 


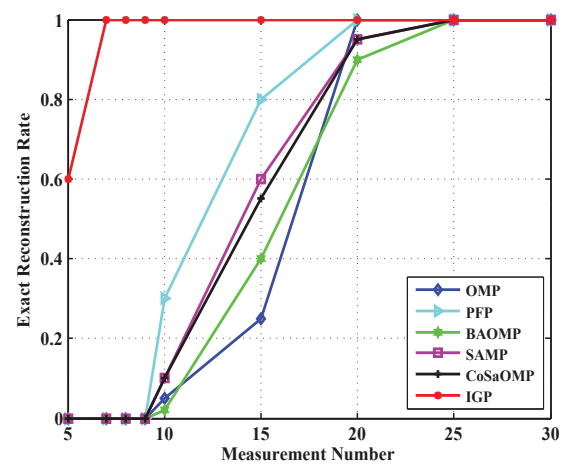

(a)

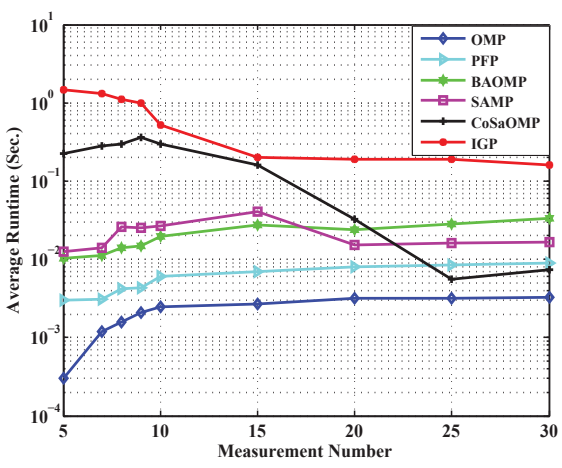

(b)

Figure 1: Reconstruction performance of IGP, OMP, PFP, BAOMP, SAMP and CoSaOMP with the measurement number changing from 5 to 30 for the fixed $N=50, K=5$. a) Exact Reconstruction Rate, b) Average Runtime.

Secondly, we fix $N=50$ and $m=20$ and change the sparsity level $K$ from 0 to 14. The simulation results of the six methods are shown in Fig.2. As we can see from Fig.2.(a), IGP performs best with the sparsity level changing from 0 to 14. In addition, IGP achieves significant improvement when the sparsity level is relatively large. Fig.2.(b) shows that the average runtime of IGP is larger than the state-of-the-art greedy algorithms with relatively high sparsity level but smaller than SAMP, BAOMP, PFP and CoSaOMP when the sparsity level is relatively small.

Thirdly, we fix $K=5$ and $m=20$ and change the length of the original signal from 50 to 200. The results of the six methods are shown in Fig.3. We also see from Fig.3.(a) that IGP has the best performance for all the signal length, especially when the signal length is relatively small. Fig.3.(b) shows that the reconstruction speed of IGP is slower than the state-of-the-art methods. 


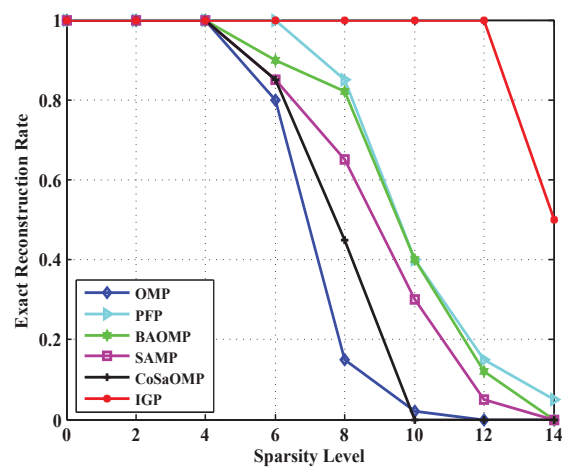

(a)

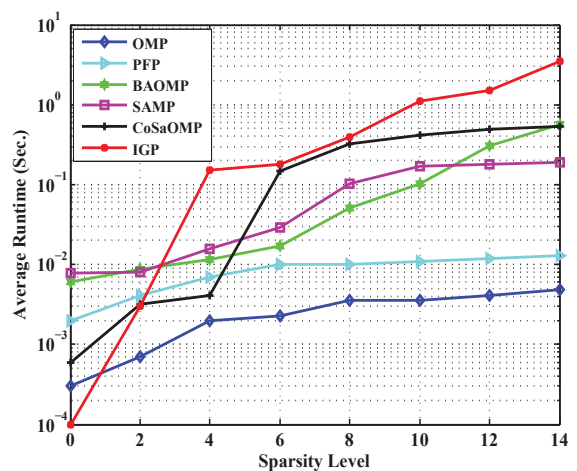

(b)

Figure 2: Reconstruction performance of IGP, OMP, PFP, BAOMP, SAMP and CoSaOMP with the sparsity level changing from 0 to 14 for the fixed $N=50$, $m=20$. a) Exact Reconstruction Rate, b) Average Runtime.

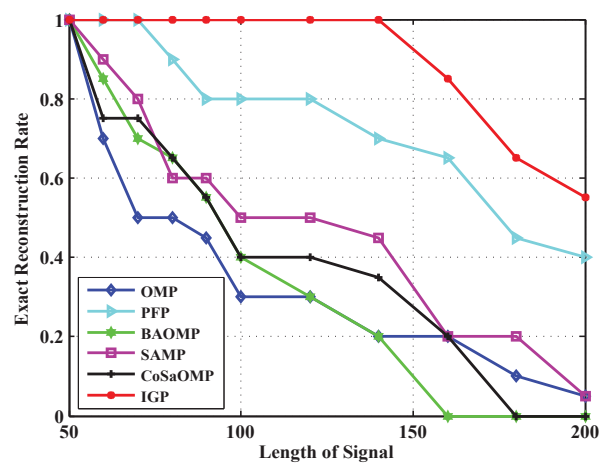

(a)

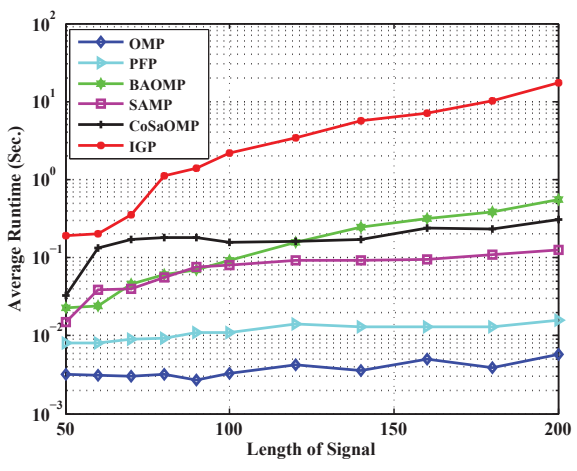

(b)

Figure 3: Reconstruction performance of IGP, OMP, PFP, BAOMP, SAMP and CoSaOMP with the length of signal changing from 50 to 200 for the fixed $m=20$, $K=5$. a) Exact Reconstruction Rate, b) Average Runtime.

The IGP method aims at solving $l_{0}$ minimization essentially by estimating the collection which contains the support collection of the original signal intelligently and accurately. Based on the superior performance of the two-cycle optimization algorithm in global searching, IGP is more likely to find the global optimal solution. If the number of iterations is relatively large, IGP can find the 


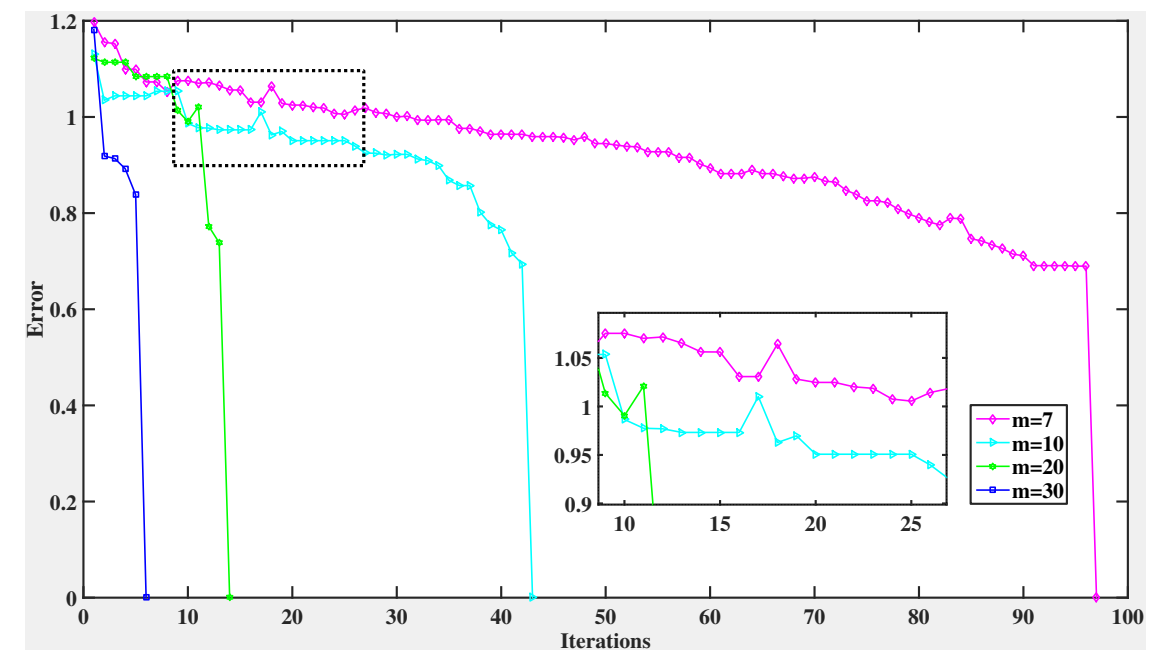

Figure 4: The number of iterations of IGP when the measurement number is set as $m=7, m=10, m=20$ and $m=30$ respectively with the fixed $N=50$ and $K=5$.

global solution with a relatively small measurement number. Considering the reconstruction speed, it is influenced significantly by the iterations. Fig.4 shows the iterations when the measurement number is set as $m=7, m=10, m=20$ and $m=30$ respectively with the fixed $N=50$ and $K=5$. We find that the number of iterations reduces with the measurement number increasing, so the reconstruction speed of IGP performs better when the measurement number is relatively large or the sparsity level is relatively small. The main reason of the singular point in the enlarged area marked by the black box in Fig.4 is that the two-cycle method permits accepting a less optimal solution and also some random solutions with positive probability, which can avoid IGP method falling into a sub-optimal solution. As the size of the solution set can be calculated by $C_{N}^{K}$, it becomes big with the increasing sparsity level or the increasing signal length, which leads to a relatively larger number of iterations than greedy algorithm. Although the estimation principle is designed by matching strategies of greedy algorithm, the reconstruction speed is slower than the greedy algorithms in many cases. This analysis can also be illustrated in Fig.1, Fig.2 and 
Fig.3. In conclusion, all the simulation results show that IGP outperforms the state-of-the-art algorithms in reconstruction quality and the average runtime of IGP also performs better in some cases. Moreover, IGP can reconstruct signal accurately with a relatively small measurement number.

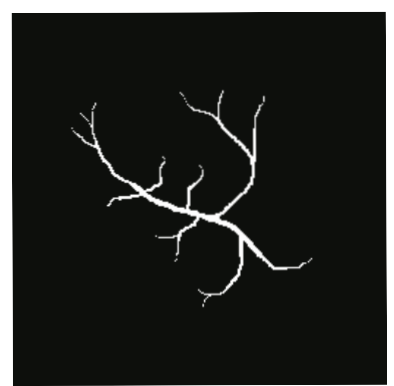

(a)

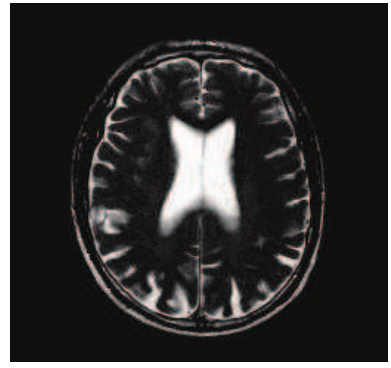

(d)

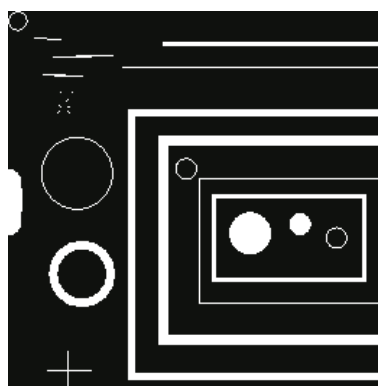

(b)

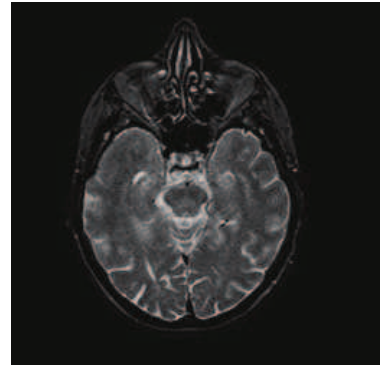

(e)

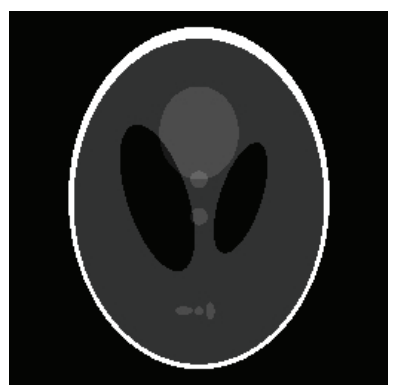

(c)

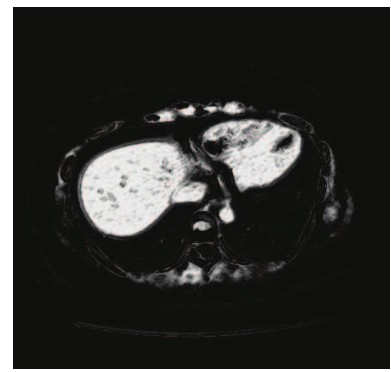

(f)

Figure 5: Test images. (a) photo-acoustic image, (b) blobs image, (c) phantom image, (d) brain MR image, (e) brain CT image, (f) liver CT image.

\subsection{Experiments on image}

In this experiment, IGP is used for image reconstruction. The test images are photo-acoustic image, blobs image, phantom image, brain MR image, brain CT image and liver CT image respectively, which are shown in Fig.5. The measurement rate of the first three images are fixed as 0.3 and that of the last three images are fixed as 0.5. To illustrate the efficiency of IGP model, we compare IGP with the state-of-the-art methods, including BP, OMP, PFP, Lasso, CoSaMP and EMPA, and evaluate the reconstruction performance by the 
simulation results of PSNR, SSIM [35], Time and visual quality. The results of PSNR, SSIM and Time is presented in Table 1 and Table 2, while the visual quality of the seven methods are shown in Fig.6, Fig.7, Fig.8 and Fig.9.

Table 1: Reconstruction performance of OMP, PFP, BP, Lasso, CoSaMP, EMPA and the proposed IGP for photo-acoustic image, blobs image and phantom image with the fixed measurement rate 0.3 .

\begin{tabular}{lcccc}
\hline Image & Methods & PSNR (dB) & SSIM & Time (Sec.) \\
\hline \multirow{4}{*}{ photo-acoustic } & OMP & 29.7484 & 0.9246 & 3.6795 \\
& PFP & 44.5281 & 0.9985 & 49.5601 \\
& BP & 22.3140 & 0.9745 & 15.1924 \\
& Lasso & 34.4204 & 0.9791 & 2.6163 \\
& CoSaMP & 73.0188 & 1.0000 & 24.7552 \\
& EMPA & 43.0942 & 0.9973 & $\mathbf{2 . 1 1 2 5}$ \\
& Proposed IGP & $\mathbf{2 7 8 . 2 3 6 5}$ & $\mathbf{1 . 0 0 0 0}$ & 15.0102 \\
& & & & \\
blobs & OMP & 23.3237 & 0.9136 & 4.4853 \\
& PFP & 24.4160 & 0.9015 & 67.7077 \\
& BP & 17.6337 & 0.7157 & 13.7897 \\
& Lasso & 32.8725 & 0.9983 & 3.5528 \\
& CoSaMP & 37.0963 & $\mathbf{1 . 0 0 0 0}$ & 521.0282 \\
& EMPA & 31.8219 & 0.9997 & $\mathbf{3 . 8 8 3 9}$ \\
& Proposed IGP & $\mathbf{5 3 . 2 0 3 1}$ & $\mathbf{1 . 0 0 0 0}$ & 21.5800 \\
& & & \\
phantom & & & \\
& OMP & 27.8826 & 0.8925 & 4.1886 \\
& PFP & 34.8763 & 0.9506 & 52.2285 \\
& BP & 33.9970 & 0.9444 & 12.5795 \\
& Lasso & 24.1798 & 0.8816 & 5.0591 \\
& CoSaMP & 31.4155 & 0.9942 & 843.1793 \\
EMPA & 39.2611 & 0.9867 & $\mathbf{3 . 8 1 5 6}$ \\
& Proposed IGP & $\mathbf{2 5 7 . 9 6 8 1}$ & $\mathbf{1 . 0 0 0 0}$ & 15.6626 \\
\hline
\end{tabular}

From Table 1 and Table 2, we can find that our IGP method achieves the best reconstruction performance in both PSNR and SSIM for all the test images. Also, we find from Table 1 and Table 2 that the reconstruction speed of IGP is slower than that of OMP, BP, Lasso and EMPA but faster than that of PFP and CoSaMP. As IGP can reconstruct signal accurately with a relatively smaller measurement number or a relatively bigger sparsity level, it outperforms the state-of-the-art algorithms in reconstruction quality when the measurement number is fixed. In addition, IGP can solve $l_{0}$ minimization essentially and can find the global optimal solution, so it significantly improves the quality of image reconstruction. The main reason of the performance on reconstruction speed is that the intelligent estimation of IGP needs more iterations, so the computa- 
Table 2: Reconstruction performance of OMP, PFP, BP, Lasso, CoSaMP, EMPA and the proposed IGP for brain MR image, Brain CT image and liver CT image with the fixed measurement rate 0.5 .

\begin{tabular}{lcccc}
\hline Image & Methods & PSNR $(\mathrm{dB})$ & SSIM & Time (Sec.) \\
\hline & OMP & 22.8036 & 0.6787 & 12.7769 \\
brain MR & PFP & 26.0979 & 0.7653 & 137.5601 \\
& BP & 26.1914 & 0.7680 & 23.1572 \\
& Lasso & 35.1660 & 0.9076 & 12.4412 \\
& EMPA & 28.0566 & 0.8621 & 649.3953 \\
& Proposed IGP & $\mathbf{4 8 . 2 4 7 1}$ & $\mathbf{1 . 0 0 0 0}$ & 120.2910 \\
& & & & \\
brain CT & OMP & 22.1034 & 0.5854 & 11.9983 \\
& PFP & 25.1167 & 0.6779 & 109.3946 \\
& BP & 25.5366 & 0.6978 & 22.7361 \\
& Lasso & 32.3305 & 0.8319 & 13.1057 \\
& CoSaMP & 31.7627 & 0.9312 & 424.5496 \\
& EMPA & 29.7933 & 0.8845 & $\mathbf{3 . 3 0 4 3}$ \\
& Proposed IGP & $\mathbf{4 6 . 8 6 4 3}$ & $\mathbf{0 . 9 9 6 3}$ & 48.2934 \\
& OMP & 28.9369 & $\mathbf{1 . 0 0 0 0}$ & 40.3513 \\
& PFP & 32.1322 & $\mathbf{1 . 0 0 0 0}$ & 451.5205 \\
& BP & 33.6136 & $\mathbf{1 . 0 0 0 0}$ & 58.6379 \\
& Lasso & 31.6488 & 0.9988 & 49.7594 \\
& CoSaMP & 28.0207 & 0.9655 & 1486.2149 \\
EMPA & 35.0608 & $\mathbf{1 . 0 0 0 0}$ & $\mathbf{8 . 8 3 9 7}$ \\
& Proposed IGP & $\mathbf{3 8 . 6 5 4 1}$ & $\mathbf{1 . 0 0 0 0}$ & 334.1973 \\
\hline \multirow{4}{*}{ CT } & & &
\end{tabular}

tional complexity of IGP is higher than some state-of-the-art algorithms such as OMP, BP and EMPA. The simulation results presented in Table 1 and Table 2 indicate that IGP improves the reconstruction accuracy significantly for all the test images and outperforms the state-of-the-art methods on reconstruction speed in some cases.

In order to verify the superior performance of the proposed method IGP for image reconstruction, Fig.6, Fig.7, Fig.8 and Fig.9 present the visual quality of the reconstructed images obtained by the test seven methods, where the odd rows represent the whole reconstructed images and the even rows represent the enlarged local parts marked by the red blocks corresponding to the whole reconstructed images. From the enlarged local parts marked by the red blocks, we can see that the compared state-of-the-art methods produce more reconstruction error, on both edge area and smooth area. Nevertheless, IGP provides the best visual. In other word, IGP outperforms the state-of-the-art methods on 
the visual quality for all the images.

\section{CONCLUSION}

This paper proposes an intelligent greedy pursuit (IGP) model to solve the $l_{0}$ minimization essentially and improve the performance of reconstruction. We first propose a novel optimization function for the sparse reconstruction and then design a two-cycle optimization algorithm which can improve the reconstruction speed of IGP and is superior in global optimization to solve the proposed optimization function. The two-cycle optimization method estimates the collection which contains the support collection intelligently and accurately by taking advantage of intelligent optimization algorithm in global searching. The principle of the estimation is designed using the matching strategies of greedy algorithm which performs quit well in reconstruction speed. The proposed IGP model is as simple as greedy algorithm, while it overcomes the shortcomings of greedy algorithm. According to the simulation results, we can claim that the proposed IGP model outperforms the state-of-the-art algorithms in reconstruction accuracy and can reconstruct signal accurately with a relatively smaller measurement number. One possible extension of our work would be to develop some effective updating strategies to IGP to accelerate the reconstruction speed and make it suitable for the signal with a larger length, such as the so-called big data.

\section{ACKNOWLEDGMENT}

This work is financially supported by National Science Foundations of China (No.61174016) and (No.61171197). 


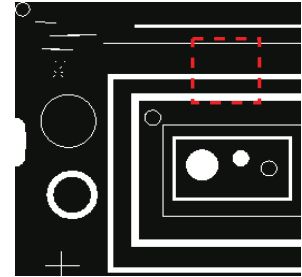

(a)
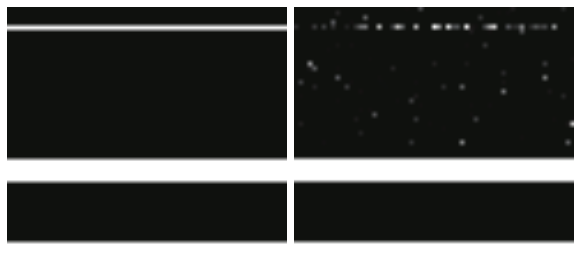

(a

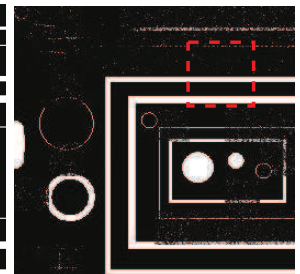

(b) (b

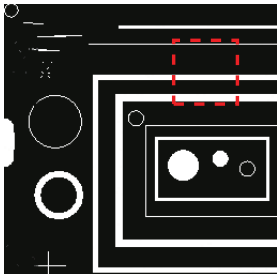

(c)

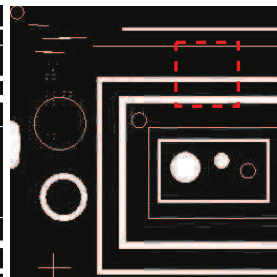

(d)
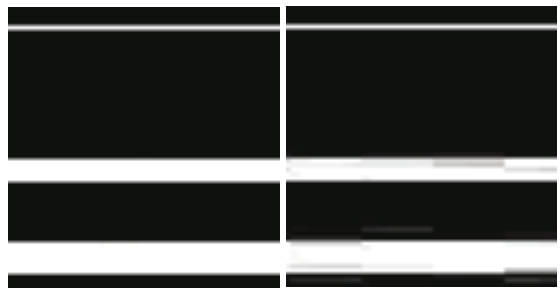

(d

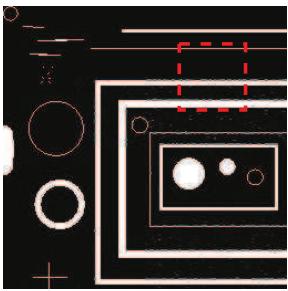

(e)

(c

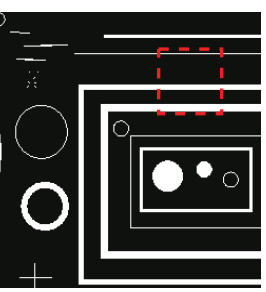

(f)
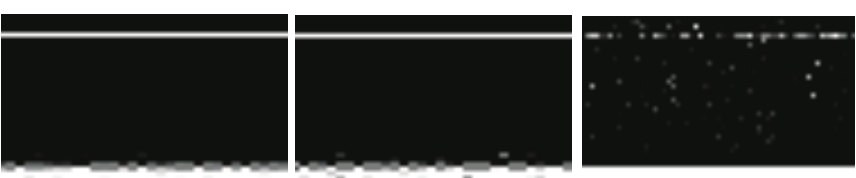

(
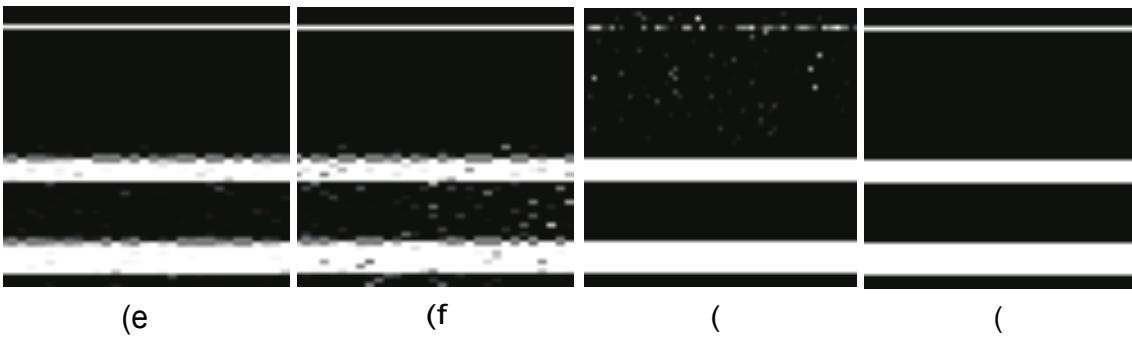

(

Figure 6: Visual quality of BP, CoSaMP, EMPA, Lasso, OMP, PFP and the proposed IGP for blobs image. The odd rows represent the whole reconstructed images obtained by the seven methods. The even rows represent the enlarged local parts marked by the red blocks corresponding to the whole images. 


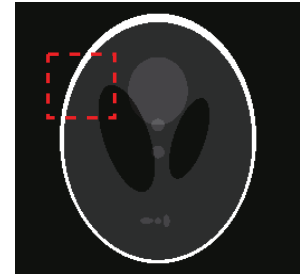

(a)

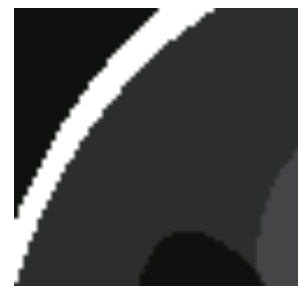

(a')

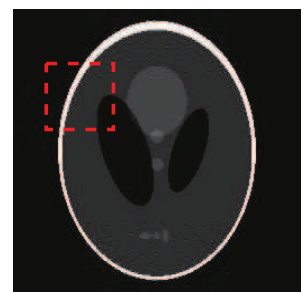

(e)

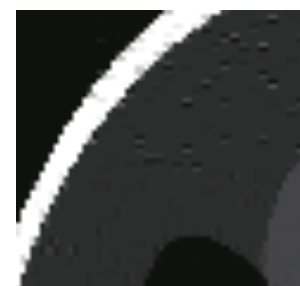

(e')

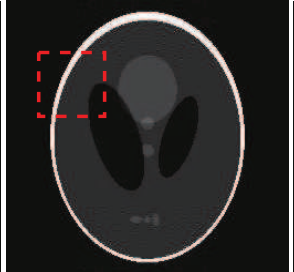

(b)

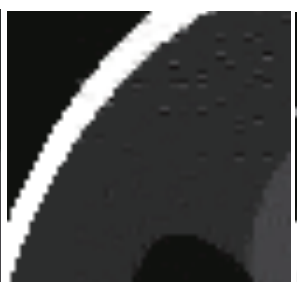

(b')

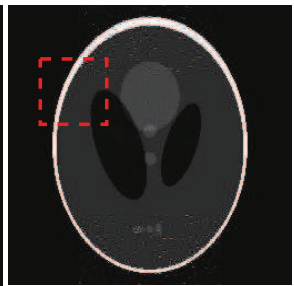

(f)

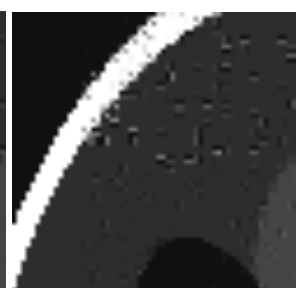

(f')

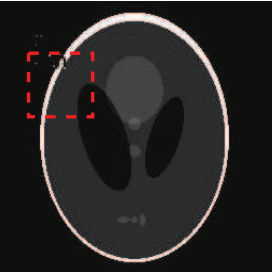

(c)

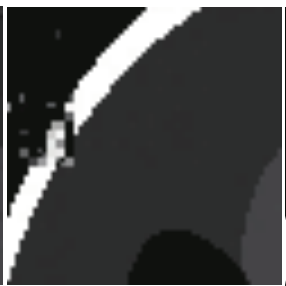

(c')

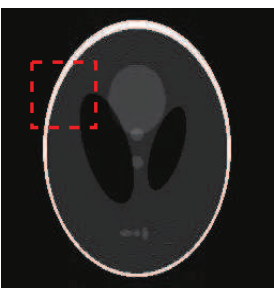

(g)

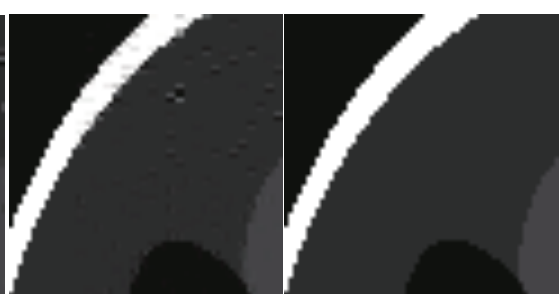

(g')

Figure 7: Visual quality of BP, CoSaMP, EMPA, Lasso, OMP, PFP and the proposed IGP for phantom image. The odd rows represent the whole reconstructed images obtained by the seven methods. The even rows represent the enlarged local parts marked by the red blocks corresponding to the whole images. 


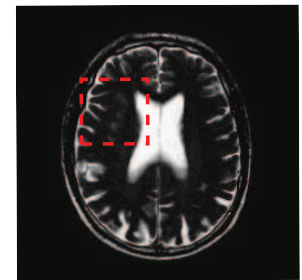

(a)

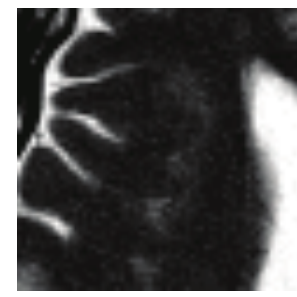

(a')

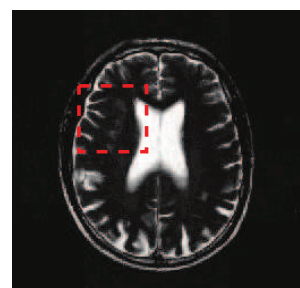

(e)

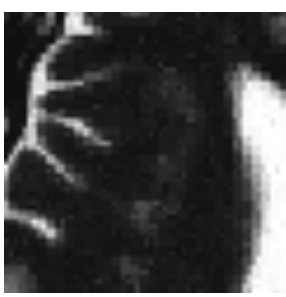

(e')

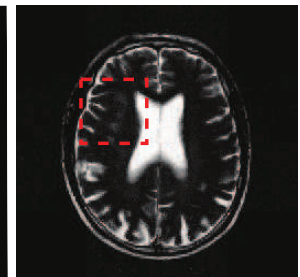

(b)

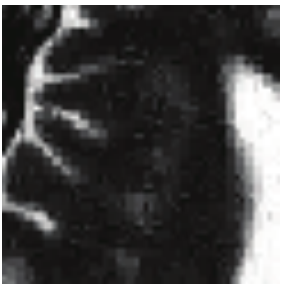

(b')

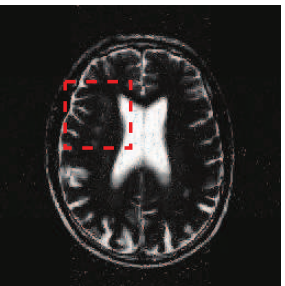

(f)

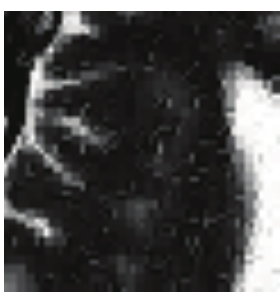

(f')

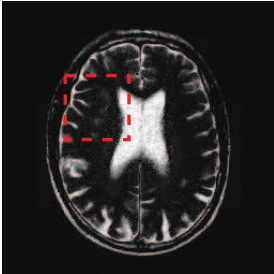

(c)

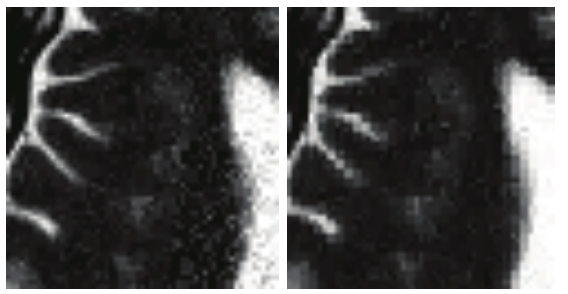

(c')

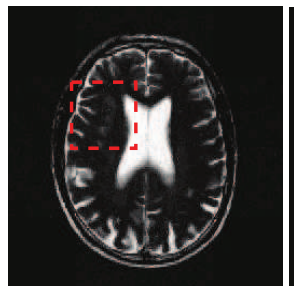

(g)

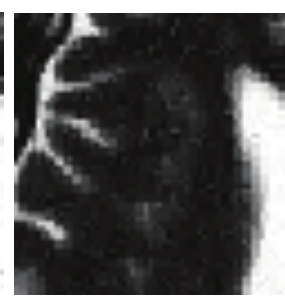

(g')

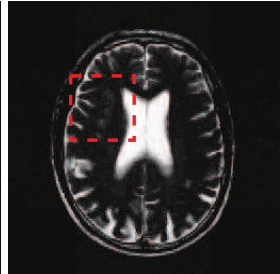

(d)

$\left(d^{\prime}\right)$

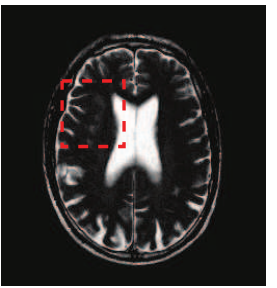

(h)

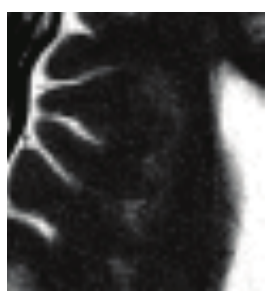

(h')

Figure 8: Visual quality of BP, CoSaMP, EMPA, Lasso, OMP, PFP and the proposed IGP for brain MR image. The odd rows represent the whole reconstructed images obtained by the seven methods. The even rows represent the enlarged local parts marked by the red blocks corresponding to the whole images. 


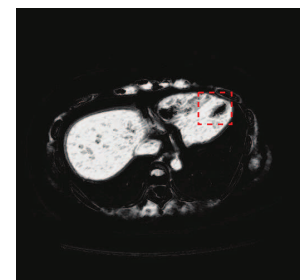

(a)

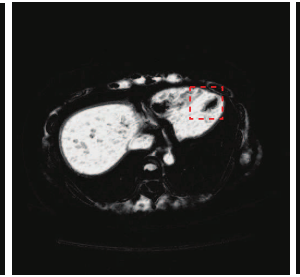

(b)

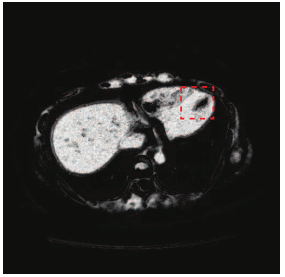

(c)

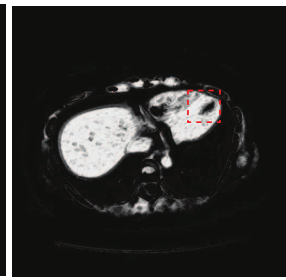

(d)

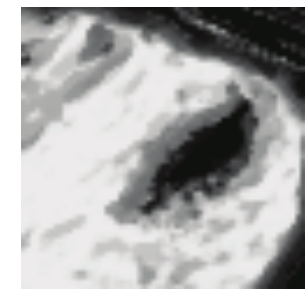

$\left(a^{\prime}\right)$

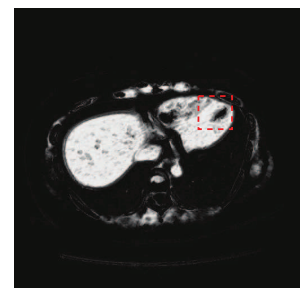

(e)

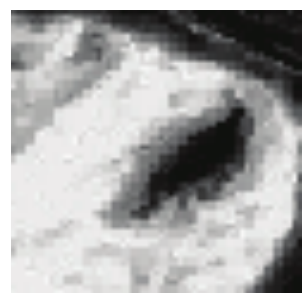

(e')

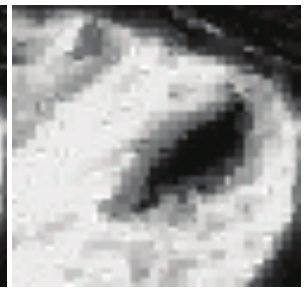

(b')

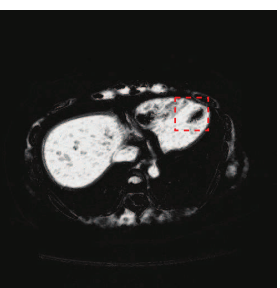

(f)

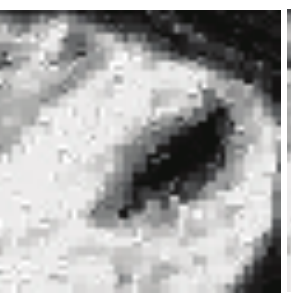

(f')

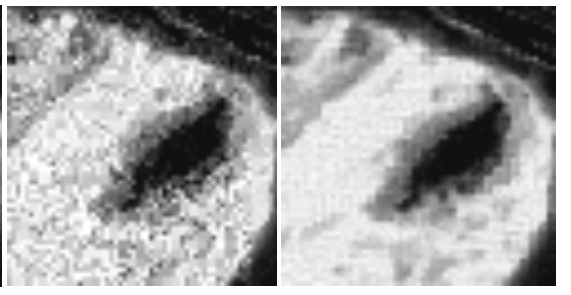

(c')

(d')

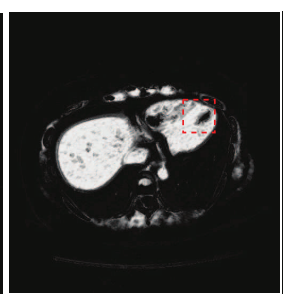

(g)

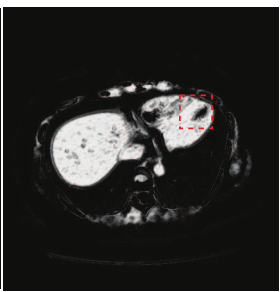

(h)

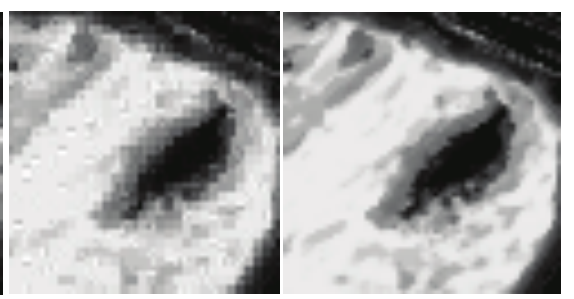

(g')

(h')

Figure 9: Visual quality of BP, CoSaMP, EMPA, Lasso, OMP, PFP and the proposed IGP for liver $\mathrm{CT}$ image. The odd rows represent the whole reconstructed images obtained by the seven methods. The even rows represent the enlarged local parts marked by the red blocks corresponding to the whole images. 


\section{References}

[1] D. L. Donoho, Compressed sensing, Information Theory, IEEE Transactions on 52 (4) (2006) 1289-1306.

[2] E. J. Candès, J. Romberg, T. Tao, Robust uncertainty principles: Exact signal reconstruction from highly incomplete frequency information, Information Theory, IEEE Transactions on 52 (2) (2006) 489-509.

[3] P.-Y. Chen, I. W. Selesnick, Group-sparse signal denoising: non-convex regularization, convex optimization, Signal Processing, IEEE Transactions on 62 (13) (2014) 3464-3478.

[4] J. Romberg, Imaging via compressive sampling [introduction to compressive sampling and recovery via convex programming], IEEE Signal Processing Magazine 25 (2) (2008) 14-20.

[5] D. L. Donoho, M. Elad, On the stability of the basis pursuit in the presence of noise, Signal Processing 86 (3) (2006) 511-532.

[6] S. S. Chen, D. L. Donoho, M. A. Saunders, Atomic decomposition by basis pursuit, SIAM journal on scientific computing 20 (1) (1998) 33-61.

[7] E. J. Candes, T. Tao, Decoding by linear programming, Information Theory, IEEE Transactions on 51 (12) (2005) 4203-4215.

[8] J. Tropp, A. C. Gilbert, et al., Signal recovery from random measurements via orthogonal matching pursuit, Information Theory, IEEE Transactions on 53 (12) (2007) 4655-4666.

[9] T. T. Cai, L. Wang, Orthogonal matching pursuit for sparse signal recovery with noise, Information Theory, IEEE Transactions on 57 (7) (2011) 46804688 .

[10] W. Dai, O. Milenkovic, Subspace pursuit for compressive sensing signal reconstruction, Information Theory, IEEE Transactions on 55 (5) (2009) $2230-2249$. 
[11] M. D. Plumbley, Recovery of sparse representations by polytope faces pursuit, in: Independent Component Analysis and Blind Signal Separation, Springer, 2006, pp. 206-213.

[12] D. Needell, J. A. Tropp, Cosamp: Iterative signal recovery from incomplete and inaccurate samples, Applied and Computational Harmonic Analysis 26 (3) (2009) 301-321.

[13] W. Yin, S. Osher, D. Goldfarb, J. Darbon, Bregman iterative algorithms for \ell_1-minimization with applications to compressed sensing, SIAM Journal on Imaging Sciences 1 (1) (2008) 143-168.

[14] H. Huang, A. Makur, Backtracking-based matching pursuit method for sparse signal reconstruction, Signal Processing Letters, IEEE 18 (7) (2011) 391-394.

[15] T. T. Do, L. Gan, N. Nguyen, T. D. Tran, Sparsity adaptive matching pursuit algorithm for practical compressed sensing, in: Signals, Systems and Computers, 2008 42nd Asilomar Conference on, IEEE, 2008, pp. 581587.

[16] D. L. Donoho, Y. Tsaig, I. Drori, J.-L. Starck, Sparse solution of underdetermined systems of linear equations by stagewise orthogonal matching pursuit, Information Theory, IEEE Transactions on 58 (2) (2012) 10941121.

[17] M. Tuba, N. Bacanin, Improved seeker optimization algorithm hybridized with firefly algorithm for constrained optimization problems, Neurocomputing 143 (2014) 197-207.

[18] B. Hajek, Cooling schedules for optimal annealing, Mathematics of operations research 13 (2) (1988) 311-329.

[19] C.-H. Lin, A rough penalty genetic algorithm for constrained optimization, Information Sciences 241 (2013) 119-137. 
[20] X. Du, L. Cheng, D. Chen, A simulated annealing algorithm for sparse recovery by 10 minimization, Neurocomputing 131 (2014) 98-104.

[21] X. Du, L. Cheng, G. Cheng, A heuristic search algorithm for the multiple measurement vectors problem, Signal Processing 100 (2014) 1-8.

[22] L. Li, X. Yao, R. Stolkin, M. Gong, S. He, An evolutionary multiobjective approach to sparse reconstruction, Evolutionary Computation, IEEE Transactions on 18 (6) (2014) 827-845.

[23] Z. Lin, Image adaptive recovery based on compressive sensing and genetic algorithm, in: Computer Science and Automation Engineering (CSAE), 2012 IEEE International Conference on, Vol. 2, IEEE, 2012, pp. 346-349.

[24] D. Li, M. Li, Y. Shen, Y. Wang, Q. Wang, Ga-bfo based signal reconstruction for compressive sensing, in: Information and Automation (ICIA), 2013 IEEE International Conference on, IEEE, 2013, pp. 1023-1028.

[25] D. Li, C. Shi, Q. Wang, Y. Shen, Y. Wang, Artificial immune algorith$\mathrm{m}$ based signal reconstruction for compressive sensing, in: Instrumentation and Measurement Technology Conference (I2MTC) Proceedings, 2014 IEEE International, IEEE, 2014, pp. 76-81.

[26] J. W. Pepper, B. L. Golden, E. Wasil, et al., Solving the traveling salesman problem with annealing-based heuristics: a computational study, Systems, Man and Cybernetics, Part A: Systems and Humans, IEEE Transactions on 32 (1) (2002) 72-77.

[27] M. Dorigo, L. M. Gambardella, Ant colony system: a cooperative learning approach to the traveling salesman problem, Evolutionary Computation, IEEE Transactions on 1 (1) (1997) 53-66.

[28] X. Fengmin, W. Shanhe, A hybrid simulated annealing thresholding algorithm for compressed sensing, Signal Processing 93 (6) (2013) 1577-1585. 
[29] P. Pal, P. P. Vaidyanathan, On application of lasso for sparse support recovery with imperfect correlation awareness, in: Signals, Systems and Computers (ASILOMAR), 2012 Conference Record of the Forty Sixth Asilomar Conference on, IEEE, 2012, pp. 958-962.

[30] C. La, M. N. Do, Tree-based orthogonal matching pursuit algorithm for signal reconstruction, in: Image Processing, 2006 IEEE International Conference on, IEEE, 2006, pp. 1277-1280.

[31] J. Wu, F. Liu, L. Jiao, X. Wang, B. Hou, Multivariate compressive sensing for image reconstruction in the wavelet domain: using scale mixture models, Image Processing, IEEE Transactions on 20 (12) (2011) 3483-3494.

[32] M. Stojnic, F. Parvaresh, B. Hassibi, On the reconstruction of block-sparse signals with an optimal number of measurements, Signal Processing, IEEE Transactions on 57 (8) (2009) 3075-3085.

[33] J. J. Zhao, J. X. Sun, S. L. Zhou, L. Hu, Imaging of transmission equipment based on block compressed sensing, in: Applied Mechanics and Materials, Vol. 190, Trans Tech Publ, 2012, pp. 998-1001.

[34] D. Needell, Topics in compressed sensing, arXiv preprint arXiv:0905.4482.

[35] Z. Wang, A. C. Bovik, H. R. Sheikh, E. P. Simoncelli, Image quality assessment: from error visibility to structural similarity, Image Processing, IEEE Transactions on 13 (4) (2004) 600-612. 